\title{
Normalized Time
}

National Cancer Institute

\section{Source}

National Cancer Institute. Normalized Time. NCI Thesaurus. Code C94943.

The integrated plasma concentration Cp of a tracer divided by the value of this concentration at the end of the integration time. 